\title{
Practical Tips of English Expressions for Non-Native English-Speaking Peer Reviewers
}

\author{
Mikyoung Lee ${ }^{1,2,3}$ \\ ${ }^{1}$ Nursing Department, Kwangju Women's University, Gwangju, Korea, ${ }^{2}$ Department of Educational Psychology, University of Munich, \\ Munich, Germany, ${ }^{3}$ Academic Consultant \& Trainer, Editage, Seoul, Korea
}

Peer review is an essential part in ensuring the quality of papers published in scientific journals. Good reviews are beneficial for both authors and journal editors by providing authors with the opportunity to improve their manuscripts and editors with valuable comments to aid themselves to make their decisions. Despite a plethora of research articles on general guidelines for peer reviewing, it is difficult to find papers on English expressions peer reviewers can use, in particular for nonnative English-speaking peer reviewers. Therefore, this article provides "downto-earth" guidance for non-native English-speaking reviewers to construct better quality reviews. To this end, I suggest useful English expressions to help peer reviewers, whose mother tongue is not English, enhance the quality of their reviews.

Key Words: Peer review, English expression
Received June 23, 2021

Revised July 2, 2021

Accepted July 5, 2021

Published on July 20, 2021

Corresponding author: Mikyoung lee Nursing Department, Kwangju Women's University, 40 Gwangjuyeodai-gil, Gwangsan-gu, Gwangju 62396, Korea Tel: 82-62-950-3807

Fax: 82-62-950-395

E-mail: mikylee@kwu.ac.kr https://orcid.org/0000-0001-7219-659X

Copyright (c) 2021 The Korean Society for Vascular Surgery

This is an Open Access article distributed under the terms of the Creative Commons Attribution Non-Commercial License (http://creativecommons.org/licenses/by-nc/4.0) which permits unrestricted non-commercial use, distribution, and reproduction in any medium, provided the original work is properly cited.

Cite this article; Vasc Specialist Int 2021. https://doi.org/10.5758/vsi.210044

\section{OVERVIEW}

"Peer review is a privilege, and an invitation to conduct such a review is a professional honor" [1]. An effective review of a manuscript is a gift for authors, editors, and the academic field [2]. Peer review is an essential part in ensuring the quality of papers published in scientific journals. Good reviews are beneficial for both authors and journal editors; authors will have the opportunity to improve their manuscripts in terms of quality, clarity, and accuracy, and editors will receive valuable comments to aid themselves to make their decisions on publication of manuscripts [3].

There have been a plethora of research articles on general guidelines for the peer review [2-6]. However, it is difficult to find papers on what kind of English expressions peer reviewers can use, in particular for non-native Englishspeaking reviewers. Writing poor reviews could be damaging to peer reviewers because they might not be invited to review manuscripts again and even might be evaluated as low quality reviewers in some journals [7]. Good reviews should contain not only constructive content but also appropriate English expressions so that authors and editors can clearly understand what the reviewers mean to describe. For non-native English-speaking peer reviewers, however, English writing is another substantial obstacle in providing good reviews on top of the review content itself. They need to make an extra effort to hone their English skills to write good reviews in English. Therefore, the aim of this contribution is to provide "down-to-earth" guidance for non-native English-speaking reviewers to construct better quality reviews. To this end, 1 , as an academic researcher and a formal English teaching professional myself, suggest useful English expressions to help peer reviewers, whose mother tongue is not English, enhance the quality of their reviews.

\section{FRAMEWORK OF REVIEWER COMMENTS}

To begin with, let us briefly review some considerations for reviewers when deciding whether to review a manu- 
script suggested by an editor $[2,5,8]$. First of all, reviewers should assess if they have time to complete the review within the requested time frame. If they are too busy with other obligations, they should decline the opportunity and might suggest alternative reviewers. Second, reviewers should assure that they have professional expertise in the manuscript research field. If they are not familiar enough with the subject, they should not accept the review invitation. Finally, reviewers should check whether they have a conflict of interest with authors of the manuscript or the manuscript itself. If there exists some sort of relationships in reviewing the manuscript, they should turn down the invitation; otherwise, this will prevent fair, impartial, and objective reviews.

PLOS introduces a framework for organizing reviewer comments in an effective way [9]. In the first section, reviewers should provide the summary of the research and overall impression, which is regarded as the most important information. In the second section, reviewers should demonstrate evidence and examples to support their summary provided in the first section by separating the review into major and minor issues. In the last section, they can mention any additional points about the manuscript needing improvement. In the following 1 would like to explain more details in each part of the framework and suggest example sentences that non-native English-speaking reviewers can practically use in their reviews.

\section{ENGLISH EXPRESSIONS ACCORDING TO THE FRAMEWORK}

\section{1) First section: Summary of the research and overall impression}

The most important information should be included in the first section of the summary of the research and reviewers' overall impression. Reviewers should summarize what the authors of the manuscript claim to inform. This summary will present to the editors how peer reviewers understood the manuscript and emphasize any major differences in perspectives among the reviewers. It will identify the study's strengths and weaknesses, which would be reviewers' "take-home message" for the editors [9]. To this end, reviewers should above all explicitly discuss what the main contribution of the study is; this can be done in a few sentences about the study topic [10]. Briefly starting with this will inform both the editors and the authors of what reviewers understood about the paper. Some useful sample sentences are as follows:

"This paper discusses.... The main contribution of the paper is..." [10]
"This paper represents a major effort to test the efficacy of duct tape in the treatment of warts. The methodology of the study consists of a randomized trial. The study was carried out in primary school children. The major finding was that duct tape was not better than placebo. Only one study has been previously published on this topic which was methodologically flawed." [3]

"The main strengths of this paper are that it addresses an interesting and timely question... and provides a clear answer. As such this article represents an excellent and elegant bioinformatics genome-wide study which will almost certainly influence our thinking about protein aggregation and evolution." [11]

"Some of the weaknesses are the not always easy readability of the text which establishes unclear logical links between concepts." [11]

"Thank you for the opportunity to review your work. There are some minor revisions need. The abstract needs attention. The first sentence of the abstract seems to repeat content. Recommend revising. The results component of the abstract also seems to repeat information. The description of the procedure should be sufficient to reproduce the study..." (anonymous reviewer)

"Most comments below are not criticisms to be addressed absolutely but mere suggestions for improvement, and 1 trust the authors to know better than 1 which ones are valuable to follow." (anonymous reviewer)

"This review provides a synthesis of current knowledge on.... The writing is clear and the structure is satisfying. $I$ found the paper an approachable yet in-depth review." (anonymous reviewer)

"My main comments on each of the three aspects of the paper:

1) An up-to-date database of animals... is very welcome.

2) The discussion of the various methods used...is useful, and will surely help readers interpret present data more accurately and carefully, and researchers improve their experimental protocols.

3) The discussion of the patterns identified is interesting...it could be enriched by discussing a few more perspectives." (anonymous reviewer)

After describing the valuable contribution of the study, reviewers then should give their recommendation for acceptance, minor revision, major revision or rejection. Here, reviewers should keep in mind that they are not making the decision on acceptance or rejection of the manuscript; in fact, they are only giving advice to editors, and editors are the ones who ultimately decide whether to accept the manuscript or not [1]. They can utilize one of the following sentences for the recommendation [10]: 
"I recommend that this paper be accepted."

"I recommend that this paper be accepted after minor revision."

"I recommend that this paper not be accepted without major revision."

"I recommend that this paper be rejected."

\section{2) Second section: Evidence and examples}

In the second section, reviewers should demonstrate evidence and examples to support their comments provided in the first section by structuring this section into major and minor issues [3]. Specifically, they should provide the reasons for their recommendation as well as detailed comments on specific areas for improvement [8]. That is, by dividing this section into major and minor issues, reviewers can describe the biggest issues first and then more specific issues systematically. In addition, numbering the comments is strongly recommended, considering that it will be very helpful for the authors to follow and respond to reviewers' each comment; it will be especially helpful to the authors when the reviewers refer to specific lines, pages, paragraphs, sections, or table and figure numbers, because authors are able to know exactly which parts the reviewers are mentioning $[8,9]$.

The major issues should consist of vital points to address, and reviewers should concentrate on the fundamentals of the study. For example, they can write comments on the originality of the research, the clarity of the research questions, or the suitability of the methods [3]. Some example sentences for the major issues are introduced in the following [10]:

"The statistical analysis in this paper is suitable/unsuitable for...."

"In terms of experimental technique, this paper is conventional/novel, and so...."

"The Methods section does not clearly explain...."

"The results obtained will be useful in...."

"Some of the fundamental/recent papers in the field are not cited, among these...."

"I would like to see some discussion of the findings of the papers in relation to recent findings and developments in ...."

More example sentences from anonymous reviewers are as follows:

"It would be beneficial for a general education readership to state why this area might be important for medical education and how it links with clinical practice."

"There is insufficient evidence in the introduction to provide rationale for this study."
"It is not clear whether ethical approval was sought for this project- under the Method section...."

"Abstract presents information on key areas being measured but it does not identify how these were measured. Was this a mixed methods approach, quantitative or qualitative only?"

"Argument in the second paragraph needs to be developed. What is the evidence that...? There is limited evidence in this paragraph to support claims made."

"Overall results are given but there is no information on the significance of the results."

"No explanation on design and methodological approach for the study. This needs to be explained first before describing sample and procedure."

"More detail is required on the data collection tool. How was the questionnaire constructed? How was validity and reliability of this tested questionnaire?"

In terms of the minor issues, reviewers should be aware that minor issues are still critical for improving the quality of the manuscript although they will not largely influence the main conclusions of the study [3]. Minor issues might be comprised of missing references, technical clarifications, data presentation, figures and tables, typos, spelling, grammar, phrasing issues and so on [10]. Some useful sentences from anonymous reviewers to describe minor issues are as follows:

"Page 3: Lines 38-53 this paragraph is somewhat confusing and 1 would advise rewriting it, it contains some colloquial language...."

"The list of references needs to be double checked because at least one reference had an incorrect title...."

"So of the reference source in the introduction were rather old. There are certainly more recent academic texts that would develop the concepts further."

"Page 3, paragraph 2: the notion that these data from Papp et al. convey is critical and the message needs an explicit sentence or two at end of paragraph."

"I think the title is awkward and does not do a good job of letting the reader know the true meaning of your work. 1 think the title should clue the reader about the purpose of the manuscript. Perhaps the title could be something like...."

"I think it is OK to mention the purpose on page 3 because pages 1 and 2 are an excellent example of clarification of the key study concepts. 1 found these pages to be written clearly and concisely."

"On line 86, the theoretical framework will either need some explanation or can be removed. 1 am not certain that your manuscript benefits from an explanation of the framework... a description will be very helpful." 
"Line 100, 1 think you can remove 'during the break'. I do not know if this means break between classes or holiday break. So, 1 think you can omit."

"The measurement section is a little bit lengthy. Perhaps on line 139, just say: 'all Cronbach's alphas were above 0.69."”

"The legends are too small to recognize in Fig. 2."

"On page 4, it is mentioned that..., but the study by Spencer (2010) mentions that.... Can you elaborate on this difference?"

\section{3) Last section: Additional points}

Finally, reviewers can leave any additional comments about the manuscript in addition to the summary of the research as well as major and minor issues. Some helpful example sentences are as follows:

"In several places, you've used the term..., but it seems you mean..." [10]

"Have you thought about testing this with ...?" [10]

"The findings are in line with previous work and demonstrate the importance of peer support in education. 1 would like to have learned more about how you see cognitive attribution fitting in to this model, but it isn't necessary." (anonymous reviewer)

"Try to find some areas where you can cut back on the detail but still let the reader know the quality of the measurements." (anonymous reviewer)

\section{4) Reviewing a revised manuscript}

In addition, when reviewing the revised manuscript, reviewers can refer to the following sentences excerpted from anonymous reviewers' comments.

"Thank you for submitting your paper. Overall it appears that you have addressed the main issues highlighted in the first submission."

"The revision to the background and discussion have resolved the originally identified weakness of the first submission, and therefore makes this paper more suitable for publication."

"Statistical analysis appears suitable and correct."

"The overall discussion and the final conclusion provide useful information for the readers."

"I found your edits to be very helpful in understanding your variables and outcomes."

"Your paper contributes to our knowledge of the importance of social relationships and positive emotions in the educational environment."

\section{FINAL TIPS FOR EFFECTIVE REVIEWS}

In order to provide effective feedback to authors, peer reviewers should justify their recommendations with constructive comments, concrete evidence, and specific examples [3,9]. The comments should be as specific and thorough as possible so that the authors know exactly what they need to revise to improve their manuscript. Importantly, peer reviewers should always keep in mind that they should be respectful in a writing tone. This could be also difficult for non-native English-speaking reviewers, because of the foreign language issue. It is hard to use a proper tone when it comes to English writing for them. It could be helpful for them to ask native English speakers to check whether the expressions in some of their earlier reviews are polite and also reflect cultural norms. Then reviewers will be able to write their reviews more confidently in a respectful way. Peer reviewers should be also professional in their writing, and they should remember to mention what they liked about the manuscript.

On the other hand, there are some considerations that peer reviewers should not include [9]. For instance, they should not suggest additional or unnecessary experiments that might be out of scope for the research or for the journal criteria, or take an extended period. They do not have to describe exactly how the authors should revise their manuscript; peer reviewers do not need to do work for the authors; the authors will know what they have to do to revise, and that is their obligations, not the reviewers'. Reviewers should avoid promoting their own research or hypotheses to persuade authors in their reviews. Furthermore, reviewers do not have to highlight typos and grammar mistakes in writing. If the manuscript needs considerable revision in terms of language and writing quality, reviewers can just mention this in their comments. Finally, reviewers should not submit their reviews without proofreading it; it is important for them to check everything carefully one more time before the submission.

\section{CONCLUSION}

In this article 1 briefly introduced general guidelines and suggested specific example sentences for peer reviewing. This paper is one of the first papers that provides practical English expressions for peer reviewers, whose mother tongue is not English, to construct better quality reviews. Since most journals have specific guidelines for peer reviewers, reviewers can tailor the expressions that 1 introduced in this article according to the journals that they are serving. Given that reviewers also constantly get reviews of their own papers as authors, it would be advantageous 
for non-native English-speaking reviewers to refer to how other reviewers, especially native English-speaking reviewers (we can probably tell by their affiliations and names), write their comments. This could help them compensate for the disadvantage of being non-native English speakers.

Finally, peer reviewers are at the same time authors as well. Thus, 1 personally agree with the golden rule that reviewers should deal with the manuscripts they are reviewing the same way they want to get their own manuscripts treated $[1,12]$. Therefore, reviewers should write while bearing in mind how they would feel as authors themselves if they were to receive similar reviews of their own manuscripts from other peer reviewers. This highlights that reviewers should try not to offend the authors in any way but show respect for authors by using a polite and modest tone in their comments.

\section{ACKNOWLEDGEMENTS}

1 am grateful to many anonymous reviewers whose comments were cited in this manuscript.

\section{CONFLICT OF INTEREST}

The author has nothing to disclose.

\section{ORCID}

Mikyoung Lee
https://orcid.org/0000-0001-7219-659X
1) Hill JA. How to review a manuscript. J Electrocardiol 2016;49:109-111.

2) Roberts LW, Coverdale J, Edenharder K, Louie A. How to review a manuscript: a "down-to-earth" approach. Acad Psychiatry 2004;28:81-87.

3) Spigt M, Arts IC. How to review a manuscript. J Clin Epidemiol 2010;63:1385-1390.

4) Bornmann L, Daniel HD. The manuscript reviewing process: empirical research on review requests, review sequences, and decision rules in peer review. Libr lnf Sci Res 2010;32:5-12.

5) Genter MB. How to review a manuscript: tips and suggestions. Int J Toxicol 2020;39:263.

6) Raniga SB. Decline to review a manuscript: insight and implications for AJR reviewers, authors, and editorial staff. AJR Am J Roentgenol 2020;214:723-726.

7) van Rooyen S, Black N, Godlee F. Development of the review quality instrument (RQI) for assessing peer reviews of manuscripts. J Clin Epidemiol 1999;52:625-629.

8) Brazeau GA, Dipiro JT, Fincham JE, Boucher BA, Tracy TS. Your role and responsibilities in the manuscript peer review process. Am J Pharm Educ 2008;72:69.

9) PLOS. How to write a peer review [Internet]. San Francisco: PLOS; [cited 2021 Jun 10]. Available from: https:// plos.org/resource/how-to-write-a- peer-review/.

10) Musick C, Jones C. Giving an effective peer review: sample framework and comments [Internet]. Tokyo: ThinkSCIENCE; C 2006-2021 [cited 2021 Jun 10]. Available from: https://thinkscience.co.jp/en/articles/effectivepeer-review.

11) Pastore A. Peer review examples [Internet]. London: F1000 Research; C 2012-2021 [cited 2021 Jun 10]. Available from: https://f1000research.com/ for-referees/peer-reviewing-tips/examples.

12) Min SK. Ethics and responsibilities of peer reviewers to the authors, readers, and editors. Vasc Specialist Int 2021;37:1-3. 